ANNALES

POLONICI MATHEMATICI

$99.3(2010)$

\title{
Symplectic classification of parametric complex plane curves
}

\author{
by Goo Ishikawa (Sapporo) and StanisŁaW Janeczko (Warszawa)
}

\begin{abstract}
Based on the discovery that the $\delta$-invariant is the symplectic codimension of a parametric plane curve singularity, we classify the simple and uni-modal singularities of parametric plane curves under symplectic equivalence. A new symplectic deformation theory of curve singularities is established, and the corresponding cyclic symplectic moduli spaces are reconstructed as canonical ambient spaces for the diffeomorphism moduli spaces which are no longer Hausdorff spaces.
\end{abstract}

1. Introduction. In [15], motivated by the symplectic bifurcation problem, we gave a symplectic classification of simple singularities of parametric plane curves in the real case (for the higher dimensional case, see [16]). Then we necessarily have some moduli under the classification, and to understand the moduli space well, it is natural to consider the complex analytic case. In this paper, we classify symplectically general parametric plane curve singularities and, in particular, the simple and uni-modal singularities of parametric plane curves in the complex analytic case, and completely determine the structure of symplectic moduli spaces.

An irreducible germ of a complex analytic plane curve in $\mathbb{C}^{2}$ at the origin has a normalization $f:(\mathbb{C}, 0) \rightarrow\left(\mathbb{C}^{2}, 0\right)$, which is a holomorphic parametric curve-germ. There are three natural equivalence relations among plane curvegerms: We call holomorphic parametric curve-germs $f, g:(\mathbb{C}, 0) \rightarrow\left(\mathbb{C}^{2}, 0\right)$ symplectomorphic if there exist a bi-holomorphic diffeomorphism $\sigma$ of $(\mathbb{C}, 0)$ and a bi-holomorphic symplectomorphism $\tau$ for the holomorphic symplectic form $d x \wedge d y$ on $\mathbb{C}^{2}$ satisfying $\tau(g(t))=f(\sigma(t))$. If $\sigma, \tau$ are assumed to be just bi-holomorphic diffeomorphisms (resp. homeomorphisms), then we call $f$ and $g$ diffeomorphic (resp. homeomorphic). We use the abbreviation $f \sim_{\text {symp }} g$ (resp. $f \sim_{\text {diff }} g, f \sim_{\text {homeo }} g$ ).

The following conditions on a germ of parametric holomorphic plane curve $f$ are known to be equivalent ([32], [15]):

2010 Mathematics Subject Classification: Primary 58K50; Secondary 14H20, 58D27.

Key words and phrases: symplectomorphism, moduli space, cyclic quotient singularity, Puiseux characteristic, symplectic rigidity. 
(i) $f$ has an injective representative.

(ii) $f$ is a normalization onto its image.

(iii) The diffeomorphism class of $f$ is determined by a finite jet of $f$.

(iv) The symplectomorphism class of $f$ is determined by a finite jet of $f$.

(v) The quotient vector space $\mathcal{O}_{1} / f^{*} \mathcal{O}_{2}$ is finite-dimensional.

Here $f^{*}: \mathcal{O}_{2}=\mathbb{C}\{x, y\} \rightarrow \mathcal{O}_{1}=\mathbb{C}\{t\}$ is defined by composition: $f^{*}(h)=$ $h \circ f$.

Hereafter we assume that $f$ satisfies one (and therefore all) of the above conditions.

The symplectic moduli space

$$
\mathcal{M}_{\text {symp }}(f):=\left\{g:(\mathbb{C}, 0) \rightarrow\left(\mathbb{C}^{2}, 0\right) \mid g \sim_{\text {homeo }} f\right\} / \sim_{\text {symp }}
$$

for a plane curve-germ $f:(\mathbb{C}, 0) \rightarrow\left(\mathbb{C}^{2}, 0\right)$ is the set of symplectomorphism classes of curve-germs which are homeomorphic to $f$.

The differential moduli space

$$
\mathcal{M}_{\text {diff }}(f):=\left\{g:(\mathbb{C}, 0) \rightarrow\left(\mathbb{C}^{2}, 0\right) \mid g \sim_{\text {homeo }} f\right\} / \sim_{\text {diffeo }}
$$

is the set of diffeomorphism classes of curve-germs which are homeomorphic to $f$. There is a natural surjection $\mathcal{M}_{\text {symp }}(f) \rightarrow \mathcal{M}_{\text {diff }}(f)$.

The dimension $\delta(f)=\operatorname{dim}_{\mathbb{C}} \mathcal{O}_{1} / f^{*} \mathcal{O}_{2}$ represents the number of double points appearing in a generic perturbation of $f$. The number $\delta(f)$ is equal to half of the Milnor number $\mu(f)([25], 33])$, and it also has the meaning of the symplectic codimension of $f$, that is, the number of parameters needed to produce its versal deformation via symplectomorphic equivalence ([15]). Let $F:\left(\mathbb{C} \times \mathbb{C}^{\delta}, 0\right) \rightarrow\left(\mathbb{C}^{2} \times \mathbb{C}^{\delta}, 0\right)$ be a symplectically mini-versal unfolding. Set $F(t, u)=\left(f_{u}(t), u\right)\left(u \in \mathbb{C}^{\delta}\right)$. Then we show that the symplectic moduli space of $f$ is obtained as a quotient space of a component of the $\delta$-constant locus $\left\{u \in \mathbb{C}^{\delta} \mid \delta\left(f_{u}\right)=\delta(f)\right\}$ in $\mathbb{C}^{\delta}$. See $\$ 3$ for details.

A curve-germ $f$ is called $r$-modal, for a non-negative integer $r$, if a finite number of $s$-parameter families $(0 \leq s \leq r)$ of diffeomorphism classes form a neighborhood of $f$ in the space of parametric curve-germs. A 0-modal (resp. 1-modal, 2-modal) singularity is called a simple (resp. uni-modal, bi-modal) singularity.

We classify map-germs $(\mathbb{C}, 0) \rightarrow\left(\mathbb{C}^{2}, 0\right)$ under symplectomorphic equivalence and diffeomorphic equivalence, and describe both moduli spaces, according to the modality under diffeomorphic equivalence.

V. I. Arnold initiated investigations aiming at classifying function germs with small modalities under diffeomorphic equivalence ([1]). Then, related to Arnold's classification, Bruce and Gaffney ([3]) classified the simple singularities of parametric plane curve-germs $(\mathbb{C}, 0) \rightarrow\left(\mathbb{C}^{2}, 0\right)$ into the classes $A_{2 \ell}, E_{6 \ell}, E_{6 \ell+2}, W_{12}, W_{18}$ and $W_{1,2 \ell-1}^{\#}(\ell=1,2,3, \ldots)$. See also $\$ 5$ (Theorem 5.1). 
In this paper we achieve a symplectic classification of simple singularities of parametric plane curve-germs and we find that the symplectic moduli spaces for simple singularities have cyclic quotient singularities (Table 1). Moreover we proceed to determine the symplectic moduli space for uni-modal singularities (Table 2). These results are described in Theorems 3.1, 3.10 and 4.1. Furthermore, based on the symplectic classification, we complete the differential classification of simple and uni-modal singularities (Theorem 5.1).

O. Zariski, in a series of works on equi-singularity, studied the differential classification of parametric plane curve-germs ([34]). Two curve-germs are called equi-singular if they are homeomorphic. It is known that the homeomorphism class of a plane curve-germ $f:(\mathbb{C}, 0) \rightarrow\left(\mathbb{C}^{2}, 0\right)$ is determined exactly by its Puiseux characteristic $\left(m, \beta_{1}, \ldots, \beta_{q}\right)$ ([33], see also 2 ).

The notations of singularities, for example, in Theorem 3.10 are due to Arnold (see, for instance, [1], [3]), and are actually used for equi-singular classes. For example we denote by $N_{20}$ the class $(5,6)$ and by $W_{2,2 \ell-1}^{\#}$ the class $(4,10,2 \ell+9)$.

Zariski determined in particular the differential moduli spaces for the classes $(2,2 \ell+1),(3,3 \ell+1),(4,5),(4,6,2 \ell+5),(5,6)$ and $(6,7)$ in 34 . He did not mention symplectomorphic equivalence but he used, as pre-normal forms, equi-singular mini-versal deformations, namely mini-versal deformations among equi-singular deformations. For instance, in [34, p. 68] he started the classification with

$$
x=t^{5}, \quad y=t^{6}+a_{8} t^{8}+a_{9} t^{9}+a_{14} t^{14}
$$

in the concrete classification of the case $(5,6)$, namely $N_{20}$, which is exactly the symplectic normal form given in Theorem 3.10. Nevertheless note that equi-singular mini-versal deformation is a local notion with respect to the parameter space: any neighborhood of 0 in $\mathbb{C}^{s}$ contains all diffeomorphism classes. On the other hand, symplectic normal form is a global notion: the whole affine space $\mathbb{C}^{s}$ is necessary to cover all symplectomorphism classes. The general theory of equi-singular versal deformations is given by Wahl [31] and Teissier [27], 28], 29]. Our method will give a new insight into those results on the deformation theory of curve singularities.

Clarifying the role of symplectomorphic equivalence, we have succeeded in completing Zariski's classification via modality: In fact by classification of simple singularities [3] and by Theorem 5.1, we obtain the differential classification for the equi-singular classes $(4,7),(5,7),(5,8),(4,9),(4,11)$ and $(4,10,2 \ell+9)$ which were not obtained by $\mathrm{O}$. Zariski. The case $(6,7)$ classified by him is actually bi-modal. For this case using our symplectic method we get the exact diffeomorphic classification (Theorem 5.6) and determine the corresponding symplectic moduli space (Remark 5.5). Note that recently the classification of plane branches under diffeomorphism has been developed independently in [12, [13] by another direct method. 
In $\$ 2$ we characterize simple and uni-modal singularities by means of Puiseux characteristics, explaining the basic infinitesimal method we use throughout this paper. We give a general method to obtain a symplectic pre-normal form for any equi-singular class, in Theorem 3.1 of $\S 3$. Then we classify each equi-singular class of simple and uni-modal singularities under symplectomorphic equivalence (Theorems 3.10 and 4.1 in $\$ 3$ and in $\$ 4$, and then under diffeomorphic equivalence (Theorem 5.1) in $\S 5$.

The symplectic classification of curves in a symplectic space was also started by Arnold [2]. Note that symplectically simple curves are classified in [15] in the case of plane branches and then in [19] for simple multi-germs in symplectic spaces of arbitrary dimension. Other analytic invariants (Łojasiewicz invariants, polar invariants) inspiring our investigation were studied in [9, 26] (see also [4]).

It is worth remarking that the symplectic classification of plane curves is regarded as a special and important case of the classification of Lagrangian varieties in a symplectic space, as well as of the classification of varieties under volume preserving diffeomorphisms. For the classification of Lagrangian varieties, Givental' [10] provides the foundations of the theory. We study the classification of parametric Lagrangian varieties in [18]. For the classification of hypersurfaces under volume preserving diffeomorphisms, see [20], [30]. For recent results on the classification of map-germs $\left(\mathbb{C}^{n}, 0\right) \rightarrow\left(\mathbb{C}^{p}, 0\right)$ and varieties in $\left(\mathbb{C}^{p}, 0\right)$ under volume preserving diffeomorphisms on $\left(\mathbb{C}^{p}, 0\right)$, see $[6]$. For recent results on the classification of function-germs $\left(\mathbb{C}^{n}, 0\right) \rightarrow(\mathbb{C}, 0)$ under volume preserving (isochore) diffeomorphisms on $\left(\mathbb{C}^{n}, 0\right)$, see [8].

2. Puiseux characteristics. Let $f:(\mathbb{C}, 0) \rightarrow\left(\mathbb{C}^{2}, 0\right), f(t)=(x(t), y(t))$, be a germ of holomorphic parametric plane curve. Set $m=\operatorname{ord}(f)$, the minimum of the order of $x(t)$ and that of $y(t)$ at $t=0$. Then, using a reparametrization and the symplectomorphism $(x, y) \mapsto(y,-x)$ if necessary, we see that $f$ is symplectomorphic to a germ of the form $\left(t^{m}, \sum_{k=m}^{\infty} a_{k} t^{k}\right)$. Suppose $m \geq 2$, that is, $f$ is not an immersion.

Set $\beta_{1}=\min \left\{k \mid a_{k} \neq 0, m \nmid k\right\}$ and let $e_{1}$ be the greatest common divisor of $m$ and $\beta_{1}$, and inductively set $\beta_{j}=\min \left\{k \mid a_{k} \neq 0, e_{j-1} \nmid k\right\}$, and let $e_{j}$ be the greatest common divisor of $\beta_{j}$ and $e_{j-1}, j \geq 2$. Then $e_{q-1}>1$, $e_{q}=1$ for sufficiently large $q$, and we call $\left(m=\beta_{0}, \beta_{1}, \ldots, \beta_{q}\right)$ the Puiseux characteristic of $f$. The Puiseux characteristic is a basic diffeomorphism invariant, and it determines exactly the homeomorphic equivalence class of $f\left(22\right.$, [34]). For example, setting $e_{0}=m$, we have the number of double points $\delta(f)$ described by $\delta(f)=\frac{1}{2} \sum_{j=1}^{q}\left(\beta_{j}-1\right)\left(e_{j-1}-e_{j}\right)$ ([25], 33] $)$.

First we show:

LEMMA 2.1. $f$ is symplectomorphic to a germ $\left(t^{m}, t^{\beta_{1}}+\sum_{k=\beta_{1}+1}^{\infty} b_{k} t^{k}\right)$. 
Proof. Set $\psi(x)=\sum_{k=m}^{\beta_{1}-1} a_{k} x^{k / m}$ and $\tau_{1}(x, y)=(x, y-\psi(x))$. Then $\tau_{1}(f(t))=\left(t^{m}, \sum_{k=\beta_{1}}^{\infty} a_{k} t^{k}\right)$ with $a_{\beta_{1}} \neq 0$. Define $\alpha \in \mathbb{C}^{*}=\mathbb{C} \backslash\{0\}$ by $\alpha^{m+\beta_{1}} a_{\beta_{1}}=1$, and set $\sigma(t)=\alpha t$ and $\tau_{2}(x, y)=\left(\alpha^{-m} x, \alpha^{m} y\right)$. Then $\tau_{1}, \tau_{2}$ are both symplectomorphisms and we see that $\tau_{2}\left(\tau_{1}(f(\sigma(t)))\right)$ has the required form.

Now we recall the infinitesimal method by means of vector fields, which we are going to use throughout this paper.

Let $f_{\lambda}(t)$ be a holomorphic family of plane curve-germs. We fix $\lambda$ once for all. Then, as an infinitesimal deformation of $f_{\lambda}:(\mathbb{C}, 0) \rightarrow\left(\mathbb{C}^{2}, 0\right)$, we consider a holomorphic map-germ $v:(\mathbb{C}, 0) \rightarrow T \mathbb{C}^{2}$ to the tangent bundle of $\mathbb{C}^{2}$ covering $f_{\lambda}$ via the natural projection $\pi: T \mathbb{C}^{2} \rightarrow \mathbb{C}^{2}$. We call such a mapping $v$ a vector field along $f_{\lambda}$. Then, for a given vector field $v$ along $f_{\lambda}$, we consider the equation

$$
v=t\left(f_{\lambda}\right) \xi+w\left(f_{\lambda}\right) \eta
$$

on a pair of holomorphic vector fields $\xi$ over $(\mathbb{C}, 0)$ and $\eta$ over $\left(\mathbb{C}^{2}, 0\right)$ with the conditions $\xi(0)=0, \eta(0,0)=0$. Here $t\left(f_{\lambda}\right) \xi$ is the composition of $\xi:(\mathbb{C}, 0) \rightarrow T \mathbb{C}$ with the tangent mapping $\left(f_{\lambda}\right)_{*}:\left(T \mathbb{C}, T_{0} \mathbb{C}\right) \rightarrow T \mathbb{C}^{2}$ of $f_{\lambda}$ and $w\left(f_{\lambda}\right) \eta=\left(f_{\lambda}\right)^{*} \eta=\eta\left(f_{\lambda}\right)$, the pull-back by $f_{\lambda}$ of $\eta:\left(\mathbb{C}^{2}, 0\right) \rightarrow$ $T \mathbb{C}^{2}$. We call the equation 2.1) Mather's equation of $f_{\lambda}$ for $v$ (cf. [24]). It expresses the infinitesimal condition that the infinitesimal deformation by $v$ is recovered by diffeomorphic equivalences. Note that Mather's equation is a non-homogeneous linear equation over $\mathbb{C}$.

We call $v$ solvable if Mather's equation for $v$ has a solution $(\xi, \eta)$ satisfying the required conditions $\xi(0)=0, \eta(0,0)=0$ and holomorphically depending on $\lambda$. Note that, if $v$ is solvable, then $c v(c \in \mathbb{C})$ is also solvable by $(c \xi, c \eta)$.

Suppose $f_{\lambda}(t)$ has the form

$$
f_{\lambda}(t)=\left(t^{m}, \sum_{k=\beta_{1}}^{\infty} b_{k}(\lambda) t^{k}\right) .
$$

Then we consider in particular the vector field $v$ along $f_{\lambda}$ of the form $v(t)=$ $t^{k}\left(\partial / \partial y \circ f_{\lambda}\right)$, or simply, $v(t)=\left(0, t^{k}\right)$, the infinitesimal deformation of $f_{\lambda}$ in the second component by the term $t^{k}$. Then we call $v$ solvable up to higher order terms if $v(t)=\left(t^{k}+\psi(t)\right)\left(\partial / \partial y \circ f_{\lambda}\right)=\left(0, t^{k}+\psi(t)\right)$ is solvable for some $\psi(t)$ with $\operatorname{ord}(\psi) \geq k+1$.

In the above situation, if $\eta$ can be taken to be a Hamiltonian vector field, which generates a family of symplectomorphisms by integration against the holomorphic symplectic form $d x \wedge d y$ on $\mathbb{C}^{2}$, then $v$ is called symplectically solvable (resp. symplectically solvable up to higher order terms).

In the following sections, we are going to apply our infinitesimal method to find normal forms of plane curve-germs. Before that, we characterize simple and uni-modal singularities by means of their Puiseux characteristics. 
Lemma 2.2. Let $f:(\mathbb{C}, 0) \rightarrow\left(\mathbb{C}^{2}, 0\right)$ be a curve-germ with the Puiseux characteristic $\left(m, \beta_{1}, \ldots\right)$. If $m=4$ and $\beta_{1} \geq 13$, or $m=5$ and $\beta_{1} \geq 9$, or $m \geq 6$, then the modality of $f$ is at least 2 .

Proof. First we assume $m=4, \beta_{1}=13$. Then, in any neighborhood of $f$, there exists a family of germs at 0 which are diffeomorphic to

$$
g_{\lambda, \rho}=\left(t^{4}, t^{13}+t^{14}+\lambda_{1} t^{15}+\lambda_{2} t^{19}+t^{20} \rho(t)\right)
$$

for some $\left(\lambda_{1}, \lambda_{2}\right)$ belonging to a non-empty open subset in $\mathbb{C}^{2}$ and $\rho \in \mathcal{O}_{1}$. We find this family by an infinitesimal calculation: First, for each $k \in \mathbb{N}$, we try to solve Mather's equation

$$
\left(\begin{array}{c}
0 \\
t^{k}
\end{array}\right) \equiv\left(\begin{array}{c}
4 t^{3} \xi \\
\left(13 t^{12}+\cdots\right) \xi
\end{array}\right)+\left(\begin{array}{c}
\eta_{1}\left(t^{4}, t^{13}+\cdots\right) \\
\eta_{2}\left(t^{4}, t^{13}+\cdots\right)
\end{array}\right) \bmod \left(\begin{array}{c}
0 \\
t^{k+1} \mathcal{O}_{1}
\end{array}\right),
$$

with $\xi \in \mathcal{O}_{1}$ and $\eta_{1}, \eta_{2} \in \mathcal{O}_{2}$ with $\xi(0)=0, \eta_{1}(0,0)=0, \eta_{2}(0,0)=0$. Then we see that $\left(0, t^{k}\right)$ is not solvable up to higher order terms for $k=15$ and $k=19$. Second, by a formal calculation, we verify that if $g_{\lambda, \rho}$ and $g_{\lambda^{\prime}, \rho^{\prime}}$ are diffeomorphic, then necessarily $\lambda_{1}^{\prime}=\lambda_{1}, \lambda_{2}^{\prime}=\lambda_{2}$. Then we see that if $m=4$, $\beta_{1}=13$, then the modality of $f$ is $\geq 2$. Moreover, if $m=4, \beta_{1} \geq 13$ then in any neighborhood of $f$, there exists a germ with characteristic $(4,13)$. From this observation we see that if $m=4, \beta_{1} \geq 13$, then the modality of $f$ is $\geq 2$.

In the case $m=5, \beta_{1} \geq 9$, we find that, in any neighborhood of $f$, there exists a family of germs at 0 which are diffeomorphic to

$$
g_{\lambda, \rho}=\left(t^{5}, t^{9}+t^{11}+\lambda_{1} t^{12}+\lambda_{2} t^{17}+t^{18} \rho(t)\right) .
$$

Moreover we see that if $g_{\lambda, \rho}$ and $g_{\lambda^{\prime}, \rho^{\prime}}$ are diffeomorphic, then necessarily $\lambda_{1}^{\prime}= \pm \lambda_{1}, \lambda_{2}^{\prime}=\lambda_{2}$. Thus we see that if $m=5, \beta_{1} \geq 9$, then the modality of $f$ is $\geq 2$.

For the case $m=6, \beta_{1} \geq 7$, similarly to the above, we find

$$
g_{\lambda, \rho}=\left(t^{6}, t^{7}+t^{9}+\lambda_{1} t^{10}+\lambda_{2} t^{11}+t^{12} \rho(t)\right),
$$

and if $g_{\lambda, \rho}$ and $g_{\lambda^{\prime}, \rho^{\prime}}$ are diffeomorphic, then necessarily $\lambda_{1}^{\prime}= \pm \lambda_{1}, \lambda_{2}^{\prime}=\lambda_{2}$. (A detailed classification of the case $(6,7)$ is given at the end of $\$ 5$.) Thus we see that if $m=6, \beta_{1} \geq 7$, then the modality of $f$ is $\geq 2$.

Lastly in the case $m \geq 7$, we see that in any neighborhood of $f$ there exists a germ with $m=6, \beta_{1} \geq 7$. Therefore also in this case we find that the modality of $f$ is greater than or equal to 2 .

We give an exact classification for the remaining characteristics in $\$ 5$, and, in fact, any germ with one of those Puiseux characteristics turns out to be simple or uni-modal. 
3. Symplectic normal forms. Let $f:(\mathbb{C}, 0) \rightarrow\left(\mathbb{C}^{2}, 0\right)$ be a holomorphic map-germ.

We briefly recall the theory developed in [15], which is applied to the complex analytic case: The symplectic codimension of $f$ is defined by

$$
\operatorname{sp-cod}(f)=\operatorname{dim}_{\mathbb{C}} \frac{V_{f}}{t f\left(V_{1}\right)+w f\left(V H_{2}\right)}
$$

as an infinitesimal symplectic invariant of Mather's type. Here $V_{f}$ is the space of germs of holomorphic vector fields $v:(\mathbb{C}, 0) \rightarrow T \mathbb{C}^{2}$ along $f$, which is the space of infinitesimal deformations of $f, V_{1}$ the space of germs of holomorphic vector fields over $(\mathbb{C}, 0)$ and $V_{2}$ the space of germs of holomorphic Hamiltonian vector fields over $\left(\mathbb{C}^{2}, 0\right)$. The homomorphisms $t f: V_{1} \rightarrow V_{f}$ and $w f: V H_{2} \rightarrow V_{f}$ are defined by $t f(\xi):=f_{*}(\xi), \xi \in V_{1}$ and $w f(\eta):=\eta \circ f$ respectively as in $\$ 2$. Here we assume neither $\xi(0)=0$ nor $\eta(0,0)=0$.

An unfolding $F:\left(\mathbb{C} \times \mathbb{C}^{r},(0,0)\right) \rightarrow\left(\mathbb{C}^{2} \times \mathbb{C}^{r},(0,0)\right)$ of $f, F(t, u)=$ $\left(f_{u}(t), u\right)$, is symplectically versal if $\frac{\partial f_{u}}{\partial u_{1}}(t, 0), \ldots, \frac{\partial f_{u}}{\partial u_{r}}(t, 0)$ generate $V_{f}$, over $\mathbb{C}$, up to the space $t f\left(V_{1}\right)+w f\left(V H_{2}\right)$ of deformations which are covered by symplectomorphisms ([15, Proposition 7.1]).

Set $f(t)=(x(t), y(t))$. For a vector field $v(t)=a(t) \partial / \partial x+b(t) \partial / \partial y$ along $f$, we define a generating function $e(t) \in \mathcal{O}_{1}$ of $v$ by

$$
d(e(t))=b(t) d(x(t))-a(t) d(y(t)),
$$

or $e^{\prime}(t)=b(t) x^{\prime}(t)-a(t) y^{\prime}(t)$. The generating function is determined up to a constant term. The generating function of $t f(\xi)+w f\left(X_{k}\right)$ is equal to $f^{*} k$, where $k$ is a Hamiltonian function of the Hamiltonian vector field $X_{k}$. Then there exists an exact sequence of vector spaces

$$
0 \rightarrow \frac{V_{f}^{\prime}}{t f\left(V_{1}\right)} \rightarrow \frac{V_{f}}{t f\left(V_{1}\right)+w f\left(V H_{2}\right)} \rightarrow \frac{R_{f}}{f^{*} \mathcal{O}_{2}} \rightarrow 0
$$

where

$$
R_{f}=\left\{e(t) \in \mathcal{O}_{1} \mid \operatorname{ord}\left(e^{\prime}(t)\right) \geq \operatorname{ord}(f)-1\right\}=\mathbb{C}+\mathfrak{m}_{1}^{m},
$$

where $\mathfrak{m}_{1}=\left\{a(t) \in \mathcal{O}_{1} \mid a(0)=0\right\}$, and

$$
V_{f}^{\prime}=\left\{v(t)=a(t) \partial / \partial x+b(t) \partial / \partial y \in V_{f} \mid b(t) x^{\prime}(t)-a(t) y^{\prime}(t)=0\right\} .
$$

Then we see that $\operatorname{dim}_{\mathbb{C}} V_{f}^{\prime} / t f\left(V_{1}\right)=\operatorname{ord}(f)-1=\operatorname{dim}_{\mathbb{C}} \mathcal{O}_{1} / R_{f}$. Therefore

$$
\operatorname{sp-cod}(f)=\operatorname{dim}_{\mathbb{C}} V_{f}^{\prime} / t f\left(V_{1}\right)+\operatorname{dim}_{\mathbb{C}} R_{f} / f^{*} \mathcal{O}_{2}=\operatorname{dim}_{\mathbb{C}} \mathcal{O}_{1} / f^{*} \mathcal{O}_{2}=\delta(f) .
$$

For example, the symplectically versal unfolding of the germ $f(t)=$ $\left(t^{5}, t^{6}\right)$ of type $N_{20}, \delta(f)=10$, is given by

$$
\left\{\begin{array}{l}
x=t^{5}+\mu_{1} t^{3}+\mu_{2} t^{2}+\mu_{3} t \\
y=t^{6}+\lambda_{1} t^{8}+\lambda_{2} t^{9}+\lambda_{3} t^{14}+\nu_{1} t^{4}+\nu_{2} t^{3}+\nu_{3} t^{2}+\nu_{4} t,
\end{array}\right.
$$


with ten parameters $\mu_{1}, \mu_{2}, \mu_{3}, \lambda_{1}, \lambda_{2}, \lambda_{3}, \nu_{1}, \nu_{2}, \nu_{3}, \nu_{4}$. In fact, $V_{f}^{\prime}$ is generated by the vector fields $\left(1, \frac{6}{5} t\right),\left(t, \frac{6}{5} t^{2}\right),\left(t^{2}, \frac{6}{5} t^{3}\right),\left(t^{3}, \frac{6}{5} t^{4}\right)$ modulo $t f\left(V_{1}\right)$. On the other hand, $R_{f} / f^{*} \mathcal{O}_{2}$ has the basis $t^{7}, t^{8}, t^{9}, t^{13}, t^{14}, t^{19}$. Then the vector fields $\left(0, t^{2}\right),\left(0, t^{3}\right),\left(0, t^{4}\right),\left(0, t^{8}\right),\left(0, t^{9}\right),\left(0, t^{14}\right)$ have generating functions which generate $R_{f} / f^{*} \mathcal{O}_{2}$. Therefore these ten vector fields form a basis of $V_{f}$ modulo $t f\left(V_{1}\right)+w f\left(V H_{2}\right)$. Moreover the first four vector fields can be replaced by $(0, t),(t, 0),\left(t^{2}, 0\right),\left(t^{3}, 0\right)$ modulo $t f\left(V_{1}\right)+w f\left(V H_{2}\right)$. Note that $(1,0) \in w f\left(V H_{2}\right)$. Thus we have the above symplectically versal unfolding.

The symplectically mini-versal unfolding is unique up to symplectomorphism of unfoldings.

In general, some of the parameters of the symplectically versal unfolding correspond to deformations into less singular germs, and the remaining parameters provide the symplectic normal form within a given equi-singular class up to some discrete symplectomorphism classes. For instance, in the above example, setting $\mu_{1}=\mu_{2}=\mu_{3}=0, \nu_{1}=\nu_{2}=\nu_{3}=\nu_{4}=0$, we have the symplectic normal form for $N_{20}$.

Let $f$ be of Puiseux characteristic $\left(m, \beta_{1}, \ldots, \beta_{q}\right)$. A monomial basis of $\mathcal{O}_{1} / f^{*} \mathcal{O}_{2}$ can be calculated by considering the order semigroup

$$
S(f)=\left\{\operatorname{ord}(k) \mid k \in f^{*} \mathcal{O}_{2}\right\} \subseteq \mathbb{N} .
$$

In fact $\left\{t^{r} \mid r \in \mathbb{N} \backslash S(f), r>0\right\}$ forms a monomial basis of $\mathcal{O}_{1} / f^{*} \mathcal{O}_{2}$.

We show the following general result on symplectic classification via the order semigroup:

Theorem 3.1. Let $f:(\mathbb{C}, 0) \rightarrow\left(\mathbb{C}^{2}, 0\right), f(t)=\left(t^{m}, t^{\beta_{1}}+\sum_{k=\beta_{1}+1}^{\infty} b_{k} t^{k}\right)$ be a germ of Puiseux characteristic $\left(m, \beta_{1}, \ldots, \beta_{q}\right)$. Let $r_{1}+m, \ldots$ $\ldots, r_{s}+m\left(r_{1}<\cdots<r_{s}\right)$ be all elements of $\mathbb{N} \backslash S(f)$ with $r_{j}>\beta_{1}(1 \leq j \leq s)$. Then $f$ is symplectomorphic to

$$
f_{\lambda}(t)=\left(t^{m}, t^{\beta_{1}}+\lambda_{1} t^{r_{1}}+\cdots+\lambda_{s} t^{r_{s}}\right)
$$

for some $\lambda=\left(\lambda_{1}, \ldots, \lambda_{s}\right) \in \mathbb{C}^{s}$.

Theorem 3.1 is an improvement of Zariski's result ([34, Proposition 1.2]) on the short representation of a branch.

To prove Theorem 3.1, note that a system of generators for the semigroup $S(f)$ is calculated explicitly from the Puiseux characteristic $\left(m, \beta_{1}, \ldots, \beta_{q}\right)$. Define an integer $($ for $0 \leq j<q)$

$$
\bar{\beta}_{j+1}:=\beta_{j+1}+\frac{m-e_{1}}{e_{j}} \beta_{1}+\frac{e_{1}-e_{2}}{e_{j}} \beta_{2}+\cdots+\frac{e_{j-1}-e_{j}}{e_{j}} \beta_{j} .
$$

Recall that $e_{j}$ is the greatest common divisor of $\beta_{j}$ and $e_{j-1}(j \geq 1)$, and $e_{0}=m$. Then we have 
LEMMA $3.2([33])$.

(1) The semigroup $S(f)$ is generated by $m, \bar{\beta}_{1}, \ldots, \bar{\beta}_{q}$, which is a minimal number of generators. The number $\bar{\beta}_{j}$ is the least element of $S(f)$ not divisible by $e_{j-1}$.

(2) Each $\bar{\beta}_{j}$ belongs to the additive semigroup $\left\langle\beta_{1}, \ldots, \beta_{j}\right\rangle$ generated by $\beta_{1}, \ldots, \beta_{j}(j \geq 1)$.

(3) $\bar{\beta}_{1}=\beta_{1}, \bar{\beta}_{j}>\beta_{j}+m$, and $\beta_{j}+m \notin S(f)$ for $2 \leq j \leq q$.

Proof. The proof of (1) is given in [33, Theorem 4.3.5]. The claim (2) is clear from the above definition of $\bar{\beta}_{j}$. Also the claims in (3) that $\bar{\beta}_{1}=\beta_{1}$ and $\bar{\beta}_{j}>\beta_{j}+m$ are clear. To see the rest of (3), suppose $\beta_{j}+m$ belongs to the semigroup $S(f)=\left\langle m, \bar{\beta}_{1}, \ldots, \bar{\beta}_{q}\right\rangle$. Then $\beta_{j}+m$ would belong to $\left\langle m, \bar{\beta}_{1}, \ldots, \bar{\beta}_{j-1}\right\rangle$, since $\bar{\beta}_{j}>\beta_{j}+m$. Then, by $(2), \beta_{j}+m$ would belong to $\left\langle m, \beta_{1}, \ldots, \beta_{j-1}\right\rangle \subseteq\left\langle e_{j-1}\right\rangle$. This contradicts the fact that $\beta_{j}$ is not divisible by $e_{j-1}$. Therefore we have (3).

LEMMA 3.3. Under the notation of Theorem 3.1, we have

$$
\left\{\beta_{2}, \ldots, \beta_{q}\right\} \subseteq\left\{r_{1}, \ldots, r_{s}\right\} .
$$

Proof. By Lemma 3.2, we see that $\beta_{j}+m \in \mathbb{N} \backslash S(f)(2 \leq j)$. Since $\beta_{j}>\beta_{1}$ we conclude that $\beta_{j} \in\left\{r_{1}, \ldots, r_{s}\right\}$.

We call a deformation of plane curve singularities equi-singular if the Puiseux characteristic is preserved.

For an equi-singular deformation $f_{\lambda}$ of $f$, we can take a monomial basis of $\mathcal{O}_{1} / f_{\lambda}^{*} \mathcal{O}_{2}$. Moreover there exists a number $N$ depending only on the Puiseux characteristic of $f$ such that if $\phi(t) \in \mathcal{O}_{1}$ has order $>N$, then $\phi(t) \in f^{*} \mathcal{O}_{2}$ ([33. Prop. 4.3.2]). In fact we may set $N=\max \{r \in \mathbb{N} \backslash S(f)\}$. Note that $N=\mu-1$ for the Milnor number $\mu$.

EXAMPLE 3.4. (1) $\left(W_{30}\right)$ Let $m=4, \beta_{1}=11$. Then the semigroup $S(f)$ is generated by 4 and 11 , and we have

$$
\mathbb{N} \backslash S(f)=\{1,2,3,5,6,7,9,10,13,14,17,18,21,25,29\} .
$$

A monomial basis of $\mathcal{O}_{1} / f^{*} \mathcal{O}_{2}$ is given by $t, t^{2}, t^{3}, t^{5}, t^{6}, t^{7}, t^{9}, t^{10}, t^{13}, t^{14}$, $t^{17}, t^{18}, t^{21}, t^{25}, t^{29}$. We see $r_{1}=13, r_{2}=14, r_{3}=17, r_{4}=21, r_{5}=25$ and $N=29$.

(2) $\left(W_{1,2 \ell-1}^{\#}\right)$ Let $m=4, \beta_{1}=6$ and $\beta_{2}=2 \ell+5$. Then $S(f)$ is generated by 4,6 and $2 \ell+11$. The complement $\mathbb{N} \backslash S(f)$ consists of $1,2,3,5,7,9,11$, $\ldots, 2 \ell+9,2 \ell+13$. We have $N=2 \ell+13$.

(3) $\left(W_{2,2 \ell-1}^{\#}\right)$ Let $m=4, \beta_{1}=10$ and $\beta_{2}=2 \ell+9$. Then $S(f)$ is generated by 4,10 and $2 \ell+19$. The complement $\mathbb{N} \backslash S(f)$ consists of $1,2,3,5,7,9$, $11,13,15,17,19,21,23, \ldots, 2 \ell+13,2 \ell+15,2 \ell+17,2 \ell+21,2 \ell+25$. We have $N=2 \ell+25$. 
Every equi-singular deformation of $f$ is a $\delta$-constant deformation. Conversely we have:

Lemma 3.5. Every $\delta$-constant deformation is equi-singular.

Proof. Let $f_{\lambda}:(\mathbb{C}, 0) \rightarrow\left(\mathbb{C}^{2}, 0\right)\left(\lambda \in\left(\mathbb{C}^{k}, 0\right)\right)$ be a $\delta$-constant deformation of $f_{0}=f$. Then, in a neighborhood of 0 in $\mathbb{C}^{k}$, we can take a monomial basis of $\mathcal{O}_{1} / f^{*} \mathcal{O}_{2}$, so we see that the order semigroup $S\left(f_{\lambda}\right)$ is constant and therefore $f_{\lambda}$ has the same Puiseux characteristic as $f$.

REMARK 3.6. (1) Homeomorphic plane curve-germs are connected by an equi-singular deformation. In fact the equi-singular germs form (in a finite jet space) a linear subspace with a finite number of linear subspaces deleted.

(2) In the parameter space of the symplectically versal unfolding of $f$, each $\delta$-constant stratum is a union of equi-singular strata by Lemma 3.5. different equi-singular strata cannot be connected by a $\delta$-constant deformation.

(3) Lemma 3.5 is true only for irreducible curves. It is no longer true for reducible curves.

Consider an equi-singular deformation $f_{\lambda}\left(\lambda \in \mathbb{C}^{s}\right)$ of a plane curvegerm $f$ with Puiseux characteristic $\left(m, \beta_{1}, \ldots, \beta_{q}\right)$. Suppose $f_{\lambda}(t)$ has the form

$$
f_{\lambda}(t)=\left(t^{m}, \sum_{k=\beta_{1}}^{\infty} b_{k}(\lambda) t^{k}\right) .
$$

To get normal forms under symplectomorphisms, we have to show that a vector field $v$ along $f_{\lambda}$ is symplectically solvable for Mather's equation

$$
v=t f_{\lambda}(\xi)+w f_{\lambda}(\eta),
$$

with $\xi(0)=0, \eta(0,0)=0$. Then we have to show that the generating function of $v$ belongs to $f^{*} \mathfrak{m}_{2}^{2}$, where $\mathfrak{m}_{2}^{2}=\left\{h \in \mathcal{O}_{2} \mid \operatorname{ord}(h) \geq 2\right\}$. Note that the Hamiltonian vector field $X_{h}$ with the Hamiltonian $h=h(x, y)$, where $h(0,0)=0$, satisfies the condition $X_{h}(0,0)=0$ if and only if $(\partial h / \partial x)(0,0)=$ $(\partial h / \partial y)(0,0)=0$.

Then we have the following:

Lemma 3.7. Let $N$ denote $\max \{r \in \mathbb{N} \backslash S(f)\}$. Then:

(1) For any $\psi(t)$ with $\operatorname{ord}(\psi)>N$, there exists a holomorphic function $h_{\lambda}(x, y)$ on $\left(\mathbb{C}^{2}, 0\right)$, holomorphically depending on $\lambda$ and satisfying $\psi(t)=h_{\lambda}\left(f_{\lambda}(t)\right)$ and $\operatorname{ord}\left(h_{\lambda}\right) \geq 2$.

(2) Any vector field $v=(0, \rho(t))$ along $f_{\lambda}$ is symplectically solvable if $\operatorname{ord}(\rho)>N-m$.

Proof. (1) Define $F:\left(\mathbb{C} \times \mathbb{C}^{s},(0,0)\right) \rightarrow\left(\mathbb{C}^{2} \times \mathbb{C}^{s},(0,0)\right)$ by $F(t, \lambda)=$ $\left(f_{\lambda}(t), \lambda\right)$. Then we see

$$
\mathfrak{m}_{1}^{N+1} \mathcal{O}_{1+s} \subseteq F^{*} \mathcal{O}_{2+s}+\mathfrak{m}_{s} F^{*} \mathcal{O}_{2+s}
$$


Since $F^{*} \mathcal{O}_{2+s}$ is a finite $\mathcal{O}_{s}$-module, we see $\mathfrak{m}_{1}^{N+1} \mathcal{O}_{1+s} \subseteq F^{*} \mathcal{O}_{2+s}$ by Nakayama's lemma (see for instance 24]). Suppose $\psi(t)=h_{\lambda}\left(f_{\lambda}(t)\right)$ for some $h_{\lambda}(x, y) \in \mathcal{O}_{3}$ and $\operatorname{ord}(\psi(t))>N$. Set $h_{\lambda}(x, y)=a_{\lambda}+b_{\lambda} x+c_{\lambda} y+k_{\lambda}(x, y)$ with $a_{\lambda}, b_{\lambda}, c_{\lambda} \in \mathcal{O}_{s}$, ord $\left(k_{\lambda}\right) \geq 2$. Then $\psi(t)=h_{\lambda}\left(f_{\lambda}(t)\right)=a_{\lambda}+b_{\lambda} x_{\lambda}(t)+$ $c_{\lambda} y_{\lambda}(t)+k_{\lambda}\left(f_{\lambda}(t)\right)$. Since ord $(\phi(t))>N \geq \beta_{1}>m$, we see $a_{\lambda}=b_{\lambda}=c_{\lambda}=0$. Thus ord $\left(h_{\lambda}\right) \geq 2$.

(2) If $\operatorname{ord}(\rho)>N-m$, then $\rho$ has a generating function $\psi$ of order $>N$. Then by (1), $\rho=h\left(f_{\lambda}(t)\right)$ with $\operatorname{ord}(h) \geq 2$. Then the Hamiltonian vector field $X_{h}$ satisfies $X_{h}(0,0)=0$. Hence $v-w f\left(X_{h}\right)$ belongs to $V_{f_{\lambda}}^{\prime}$. Since components of $v-w f\left(X_{h}\right)$ have order $\geq m$, we see that $v-w f\left(X_{h}\right)=t f(\xi)$ for some $\xi$ with $\xi(0)=0$. Thus $v$ is symplectically solvable.

Proof of Theorem 3.1. Consider the infinitesimal deformation $v=$ $\kappa(t)(\partial / \partial y \circ f), \kappa(t)=-\sum b_{k} t^{k}$, where the summation runs over $k$ different from $r_{1}, \ldots, r_{s}$. Then the deformation

$$
f_{u}=\left(t^{m}, t^{\beta_{1}}+\sum_{k=\beta_{1}+1}^{\infty} b_{k} t^{k}+u \kappa(t)\right)
$$

$(u \in[0,1])$ corresponding to $v$ is equi-singular. In fact, for $u \neq 1, f_{u}$ is equi-singular to $f_{0}$ by the definition of Puiseux characteristic. At $u=1$, let $\left(m, \beta_{1}, \beta_{2}^{\prime}, \ldots, \beta_{q^{\prime}}^{\prime}\right)$ be the Puiseux characteristic of $f_{1}$. Then $\beta_{2}^{\prime} \geq \beta_{2}$. By Lemma 3.3, $\beta_{2} \in\left\{r_{1}, \ldots, r_{s}\right\}$, so $\beta_{2}^{\prime}=\beta_{2}$. In the same way, $\beta_{3}^{\prime}=\beta_{3}, \ldots$ and finally $q^{\prime}=q$.

Then, by Lemma 3.7, there exist $w_{u}=\sum_{j=1}^{s} c_{j, u} t^{r_{j}}, c_{j, u} \in \mathbb{C}, \xi_{u} \in$ $V_{1}, \xi_{u}(0)=0$, and $\eta_{u} \in V H_{2}, \eta_{u}(0)=0$, smoothly depending on $u$ and satisfying $v=\left(0, w_{u}\right)+t f_{u}\left(\xi_{u}\right)+w f_{u}\left(\eta_{u}\right)$. By integrating from $u=0$ to $u=1$ we see that $f$ is symplectomorphic to

$$
f_{\lambda}(t)=\left(t^{m}, t^{\beta_{1}}+\lambda_{1} t^{r_{1}}+\cdots+\lambda_{s} t^{r_{s}}\right)
$$

for some $\lambda=\left(\lambda_{1}, \ldots, \lambda_{s}\right) \in \mathbb{C}^{s}$. Thus we have proved Theorem 3.1.

REMARK 3.8. The family $f_{\lambda}\left(\lambda \in \mathbb{C}^{s}\right)$ of Theorem 3.1 need not be equisingular on the whole $\mathbb{C}^{s}$. However, on each equi-singular domain $E$, the family $f_{\lambda}(\lambda \in E)$ gives a mini-transversal to the symplectic orbit of each $f_{\lambda}$ belonging to the space of germs equi-singular to $f_{\lambda}$.

A family $f_{\lambda}(t)\left(\lambda \in \mathbb{C}^{s}\right)$ is called a symplectic normal form for the Puiseux characteristic $\left(m, \beta_{1}, \ldots, \beta_{q}\right)$ if any plane curve-germ of Puiseux characteristic $\left(m, \beta_{1}, \ldots, \beta_{q}\right)$ is symplectomorphic to $f_{\lambda}(t)$ for some $\lambda \in \mathbb{C}^{s}$, and those $\lambda \in \mathbb{C}^{s}$ for which $f_{\lambda}$ is symplectomorphic to a given plane branch form a discrete subset in $\mathbb{C}^{s}$.

If there exists a symplectic normal form, then we have a surjective mapping $\mathbb{C}^{s} \rightarrow \mathcal{M}_{\text {symp }}(f)$ with discrete fibers. 
Actually we have the following result for the simple or uni-modal cases which we mainly study in this paper.

Proposition 3.9. Under the same notation as in Theorem 3.1, we have the following:

(1) If the Puiseux characteristic is $\left(m, \beta_{1}\right)$, then the family

$$
f_{\lambda}(t)=\left(t^{m}, t^{\beta_{1}}+\lambda_{1} t^{r_{1}}+\cdots+\lambda_{s} t^{r_{s}}\right),
$$

$\lambda=\left(\lambda_{1}, \ldots, \lambda_{s}\right) \in \mathbb{C}^{s}$, is a symplectomorphic normal form for the Puiseux characteristic $\left(m, \beta_{1}\right)$.

(2) If the Puiseux characteristic is $(4,6,2 \ell+5)$, then $s=\ell+1$ and $r_{1}=7$, $r_{2}=9, \ldots, r_{\ell-1}=2 \ell+3, r_{\ell}=2 \ell+5, r_{\ell+1}=2 \ell+7$. Inside the family $f_{c}(t)=\left(t^{4}, t^{6}+c_{1} t^{7}+c_{2} t^{9}+\cdots+c_{\ell-1} t^{2 \ell+3}+c_{\ell} t^{2 \ell+5}+c_{\ell+1} t^{2 \ell+7}\right)$, the subfamily

$$
f_{\lambda}(t)=\left(t^{4}, t^{6}+\lambda_{1} t^{2 \ell+5}+\lambda_{2} t^{2 \ell+7}\right),
$$

$\lambda=\left(\lambda_{1}, \lambda_{2}\right) \in \mathbb{C}^{2}, \lambda_{1} \neq 0$, is a symplectic normal form for the Puiseux characteristic $(4,6,2 \ell+5)$.

(3) If the Puiseux characteristic is $(4,10,2 \ell+9)$, then $s=\ell+4$ and $r_{1}=$ $11, r_{2}=13, r_{3}=15, \ldots, r_{\ell-1}=2 \ell+7, r_{\ell}=2 \ell+9, r_{\ell+1}=2 \ell+11$, $r_{\ell+2}=2 \ell+13, r_{\ell+3}=2 \ell+17, r_{\ell+4}=2 \ell+21$. Inside the family

$$
\begin{aligned}
f_{c}(t)= & \left(t^{4}, t^{10}+c_{1} t^{11}+c_{2} t^{13}+c_{3} t^{15}+\cdots+c_{\ell-1} t^{2 \ell+7}\right. \\
& \left.+c_{\ell} t^{2 \ell+9}+c_{\ell+1} t^{2 \ell+11}+c_{\ell+2} t^{2 \ell+13}+c_{\ell+3} t^{2 \ell+17}+c_{\ell+4} t^{2 \ell+21}\right),
\end{aligned}
$$

the subfamily

$$
f_{\lambda}(t)=\left(t^{4}, t^{10}+\lambda_{1} t^{2 \ell+9}+\lambda_{2} t^{2 \ell+11}+\lambda_{3} t^{2 \ell+13}+\lambda_{4} t^{2 \ell+17}+\lambda_{5} t^{2 \ell+21}\right),
$$

$\lambda=\left(\lambda_{1}, \lambda_{2}, \lambda_{3}, \lambda_{4}, \lambda_{5}\right) \in \mathbb{C}^{5}, \lambda_{1} \neq 0$, is a symplectic normal form for the Puiseux characteristic $(4,10,2 \ell+9)$.

Proof. In the case (1), by Theorem 3.1, there are no restrictions on $\lambda$ and we get the symplectic normal forms given in (1). In the case of $W_{1,2 \ell-1}^{\#}$, $f$ is symplectomorphic to

$$
f_{c}=\left(t^{4}, t^{6}+c_{1} t^{7}+\cdots+c_{\ell} t^{2 \ell+5}+c_{\ell+1} t^{2 \ell+7}\right) .
$$

Since the Puiseux characteristic of $f$ is $(4,6,2 \ell+5)$, we necessarily have $c_{1}=$ $0, \ldots, c_{\ell-1}=0$ and $c_{\ell} \neq 0$. Setting $\lambda=c_{\ell}, \mu=c_{\ell+1}$, we get the symplectic normal form as in (2). In the case of $W_{2,2 \ell-1}^{\#}, f$ is symplectomorphic to

$$
\begin{aligned}
f_{c}=\left(t^{4}, t^{10}+c_{1} t^{11}+c_{2} t^{13}+c_{3} t^{15}+\cdots+c_{\ell} t^{2 \ell+9}\right. \\
\left.+c_{\ell+1} t^{2 \ell+11}+c_{\ell+2} t^{2 \ell+13}+c_{\ell+3} t^{2 \ell+17}+c_{\ell+4} t^{2 \ell+21}\right) .
\end{aligned}
$$

Since the Puiseux characteristic of $f$ is $(4,10,2 \ell+9)$, we have $c_{1}=0, \ldots, c_{\ell-1}$ $=0$ and $c_{\ell} \neq 0$, which gives the symplectic normal form as in (3). 
Proposition 3.9 implies the following exact list of normal forms under symplectomorphic equivalence:

THEOREM 3.10. Every simple or uni-modal singularity $f:(\mathbb{C}, 0) \rightarrow$ $\left(\mathbb{C}^{2}, 0\right)$ is symplectomorphic to a germ which belongs to one of the following families (called "symplectic normal forms"):

$$
\begin{array}{ll}
A_{2 \ell}: \quad & \left(t^{2}, t^{2 \ell+1}\right), \\
E_{6 \ell}: \quad & \left(t^{3}, t^{3 \ell+1}+\sum_{j=1}^{\ell-1} \lambda_{j} t^{3(\ell+j)-1}\right), \\
E_{6 \ell+2}: \quad & \left(t^{3}, t^{3 \ell+2}+\sum_{j=1}^{\ell-1} \lambda_{j} t^{3(\ell+j)+1}\right), \\
W_{12}: \quad & \left(t^{4}, t^{5}+\lambda t^{7}\right), \\
W_{18}: \quad & \left(t^{4}, t^{7}+\lambda t^{9}+\mu t^{13}\right), \\
W_{1,2 \ell-1}^{\#}: \quad & \left(t^{4}, t^{6}+\lambda t^{2 \ell+5}+\mu t^{2 \ell+9}\right), \lambda \neq 0(\ell=1,2, \ldots), \\
N_{20}: \quad & \left(t^{5}, t^{6}+\lambda_{1} t^{8}+\lambda_{2} t^{9}+\lambda_{3} t^{14}\right), \\
N_{24}: \quad & \left(t^{5}, t^{7}+\lambda_{1} t^{8}+\lambda_{2} t^{11}+\lambda_{3} t^{13}+\lambda_{4} t^{18}\right), \\
N_{28}: \quad & \left(t^{5}, t^{8}+\lambda_{1} t^{9}+\lambda_{2} t^{12}+\lambda_{3} t^{14}+\lambda_{4} t^{17}+\lambda_{5} t^{22}\right), \\
W_{24}: \quad & \left(t^{4}, t^{9}+\lambda_{1} t^{10}+\lambda_{2} t^{11}+\lambda_{3} t^{15}+\lambda_{4} t^{19}\right), \\
W_{30}: \quad & \left(t^{4}, t^{11}+\lambda_{1} t^{13}+\lambda_{2} t^{14}+\lambda_{3} t^{17}+\lambda_{4} t^{21}+\lambda_{5} t^{25}\right), \\
W_{2,2 \ell-1}^{\#}: & \left(t^{4}, t^{10}+\lambda_{1} t^{2 \ell+9}+\lambda_{2} t^{2 \ell+11}+\lambda_{3} t^{2 \ell+13}+\lambda_{4} t^{2 \ell+17}+\lambda_{5} t^{2 \ell+21}\right), \\
& \lambda_{1} \neq 0(\ell=1,2, \ldots)
\end{array}
$$

4. Symplectic moduli spaces. The symplectic moduli spaces are determined by the following result:

TheOREM 4.1. Let $f_{\lambda}(t)=\left(t^{m}, t^{n}+\lambda_{1} t^{r_{1}}+\lambda_{2} t^{r_{2}}+\cdots+\lambda_{s} t^{r_{s}}\right)$ be one of the symplectic normal forms in Theorem 3.10. Then two curve-germs $f_{\lambda}$ and $f_{\lambda^{\prime}}$ belonging to the same family are symplectomorphic if and only if there exists an $(m+n)$ th root $\zeta \in \mathbb{C}$ of unity satisfying

$$
\lambda_{1}^{\prime}=\zeta^{r_{1}-n} \lambda_{1}, \quad \lambda_{2}^{\prime}=\zeta^{r_{2}-n} \lambda_{2}, \ldots, \lambda_{s}^{\prime}=\zeta^{r_{s}-n} \lambda_{s} .
$$

In particular each symplectic moduli space of a family is a Hausdorff space in the natural topology and it extends to a cyclic quotient space.

The symplectic moduli spaces are given in Tables 1 and 2 .

In the process of symplectic classification, we observe a kind of rigidity. Let $f_{\lambda}\left(\lambda \in \mathbb{C}^{s}\right)$ be one of the symplectic normal forms of simple or uni-modal parametric plane curve singularities. Since the symplectic normal form gives a mini-transversal to symplectic orbits in a sufficiently high order jet space, we see that each symplectomorphic equivalence class is isolated in the pa- 
rameter space $\mathbb{C}^{s}$. Moreover we have a stronger rigidity, symplectic rigidity, which implies Theorem 4.1. To see that, we need a series of conditions on non-linear symplectomorphisms, which is obtained via straightforward calculations.

Table 1. The complex symplectic moduli spaces of simple parametric plane curve singularities

\begin{tabular}{|c|c|c|c|}
\hline & Diff. normal form & Symp. normal form & Symp. moduli space \\
\hline$A_{2 \ell}$ & $\left(t^{2}, t^{2 \ell+1}\right)$ & $\left(t^{2}, t^{2 \ell+1}\right)$ & \\
\hline $\begin{array}{c}E_{6 \ell} \\
(\ell \geq 1)\end{array}$ & $\left(\begin{array}{c}\left(t^{3}, t^{3 \ell+1}+t^{3(\ell+p)+2}\right) \\
(0 \leq p \leq \ell-2) \\
\left(t^{3}, t^{3 \ell+1}\right)\end{array}\right.$ & $\left(\begin{array}{l}t^{3}, t^{3 \ell+1} \\
\left.\quad+\sum_{j=1}^{\ell-1} \lambda_{j} t^{3(\ell+j)-1}\right)\end{array}\right.$ & $\begin{array}{c}\mathbb{C}^{\ell-1} / G, G=\mathbb{Z} /(3 \ell+4) \mathbb{Z} \\
\left(\lambda_{1}, \ldots, \lambda_{\ell-1}\right) \mapsto \\
\left(\zeta \lambda_{1}, \ldots, \zeta^{3 j-2} \lambda_{j}, \ldots, \zeta^{3 \ell-5} \lambda_{\ell-1}\right) \\
\left(\zeta^{3 \ell+4}=1, \text { primitive }\right)\end{array}$ \\
\hline $\begin{array}{l}E_{6 \ell+2} \\
(\ell \geq 1)\end{array}$ & $\left(\begin{array}{c}\left(t^{3}, t^{3 \ell+2}+t^{3(\ell+p)+4}\right) \\
(0 \leq p \leq \ell-2) \\
\left(t^{3}, t^{3 \ell+2}\right)\end{array}\right.$ & $\left(\begin{array}{l}t^{3}, t^{3 \ell+2} \\
\left.\quad+\sum_{j=1}^{\ell-1} \lambda_{j} t^{3(\ell+j)+1}\right)\end{array}\right.$ & $\begin{array}{c}\mathbb{C}^{\ell-1} / G, G=\mathbb{Z} /(3 \ell+5) \mathbb{Z} \\
\left(\lambda_{1}, \ldots, \lambda_{\ell-1}\right) \mapsto \\
\left(\zeta^{2} \lambda_{1}, \ldots, \zeta^{3 j-1} \lambda_{j}, \ldots, \zeta^{3 \ell-4} \lambda_{\ell-1}\right) \\
\left(\zeta^{3 \ell+5}=1, \text { primitive }\right)\end{array}$ \\
\hline$W_{12}$ & $\begin{array}{c}\left(t^{4}, t^{5}+t^{7}\right) \\
\left(t^{4}, t^{5}\right)\end{array}$ & $\left(t^{4}, t^{5}+\lambda t^{7}\right)$ & $\begin{array}{l}\mathbb{C} / G, G=\mathbb{Z} / 9 \mathbb{Z} \\
\lambda \mapsto \zeta \lambda\left(\zeta^{9}=1\right)\end{array}$ \\
\hline$W_{18}$ & $\begin{array}{c}\left(t^{4}, t^{7}+t^{9}\right) \\
\left(t^{4}, t^{7}+t^{13}\right) \\
\left(t^{4}, t^{7}\right)\end{array}$ & $\left(t^{4}, t^{7}+\lambda t^{9}+\mu t^{13}\right)$ & $\begin{array}{c}\mathbb{C}^{2} / G, G=\mathbb{Z} / 11 \mathbb{Z} \\
(\lambda, \mu) \mapsto\left(\zeta \lambda, \zeta^{3} \mu\right)\left(\zeta^{11}=1\right)\end{array}$ \\
\hline $\begin{array}{l}W_{1,2 \ell-1}^{\#} \\
(\ell \geq 1)\end{array}$ & $\left(t^{4}, t^{6}+t^{2 \ell+5}\right)$ & $\begin{array}{c}\left(t^{4}, t^{6}+\lambda t^{2 \ell+5}\right. \\
\left.+\mu t^{2 \ell+9}\right) \\
(\lambda \neq 0)\end{array}$ & $\begin{array}{c}\left(\mathbb{C}^{*} \times \mathbb{C}\right) / G, G=\mathbb{Z} / 10 \mathbb{Z}, \\
(\lambda, \mu) \mapsto\left(\zeta^{2 \ell-1} \lambda, \zeta^{2 \ell+3} \mu\right) \\
\left(\zeta^{10}=1, \text { primitive }\right)\end{array}$ \\
\hline
\end{tabular}

Lemma 4.2. Let $\tau:\left(\mathbb{C}^{2}, 0\right) \rightarrow\left(\mathbb{C}^{2}, 0\right)$ be a symplectomorphism. Set

$$
\begin{aligned}
x \circ \tau(x, y)= & a x+b y+h_{1} x^{2}+h_{2} x y+h_{3} y^{2}+\ell_{1} x^{3}+\ell_{2} x^{2} y+\ell_{3} x y^{2}+\ell_{4} y^{3} \\
& +r_{1} x^{4}+r_{2} x^{3} y+r_{3} x^{2} y^{2}+r_{4} x y^{3}+r_{5} y^{4}+\cdots, \\
y \circ \tau(x, y)= & c x+e y+k_{1} x^{2}+k_{2} x y+k_{3} y^{2}+m_{1} x^{3}+m_{2} x^{2} y+m_{3} x y^{2} \\
& +m_{4} y^{3}+s_{1} x^{4}+s_{2} x^{3} y+s_{3} x^{2} y^{2}+s_{4} x y^{3}+s_{5} y^{4}+\cdots .
\end{aligned}
$$

Then

$$
\left|\begin{array}{ll}
a & b \\
c & e
\end{array}\right|=1, \quad\left|\begin{array}{cc}
2 h_{1} & b \\
2 k_{1} & e
\end{array}\right|+\left|\begin{array}{cc}
a & h_{2} \\
c & k_{2}
\end{array}\right|=0, \quad\left|\begin{array}{cc}
h_{2} & b \\
k_{2} & e
\end{array}\right|+\left|\begin{array}{cc}
a & 2 h_{3} \\
c & 2 k_{3}
\end{array}\right|=0,
$$


Table 2. The complex symplectic moduli spaces of uni-modal parametric plane curve singularities

\begin{tabular}{|c|c|c|}
\hline & Symp. normal form & Symp. moduli space \\
\hline$N_{20}$ & $\left(t^{5}, t^{6}+\lambda_{1} t^{8}+\lambda_{2} t^{9}+\lambda_{3} t^{14}\right)$ & $\begin{array}{c}\mathbb{C}^{3} / G, G=\mathbb{Z} / 11 \mathbb{Z} \\
\left(\lambda_{1}, \lambda_{2}, \lambda_{3}\right) \mapsto\left(\zeta^{2} \lambda_{1}, \zeta^{3} \lambda_{2}, \zeta^{8} \lambda_{3}\right) \\
\left(\zeta^{11}=1\right)\end{array}$ \\
\hline$N_{24}$ & $\left(t^{5}, t^{7}+\lambda_{1} t^{8}+\lambda_{2} t^{11}+\lambda_{3} t^{13}+\lambda_{4} t^{18}\right)$ & $\begin{array}{c}\mathbb{C}^{4} / G, G=\mathbb{Z} / 12 \mathbb{Z} \\
\left(\lambda_{1}, \lambda_{2}, \lambda_{3}, \lambda_{4}\right) \mapsto \\
\left(\zeta \lambda_{1}, \zeta^{4} \lambda_{2}, \zeta^{6} \lambda_{3}, \zeta^{11} \lambda_{4}\right) \\
\left(\zeta^{12}=1, \text { primitive }\right)\end{array}$ \\
\hline$N_{28}$ & $\begin{array}{l}\left(t^{5}, t^{8}+\lambda_{1} t^{9}+\lambda_{2} t^{12}\right. \\
\left.\quad+\lambda_{3} t^{14}+\lambda_{4} t^{17}+\lambda_{5} t^{22}\right)\end{array}$ & $\begin{array}{c}\mathbb{C}^{5} / G, G=\mathbb{Z} / 13 \mathbb{Z} \\
\left(\lambda_{1}, \lambda_{2}, \lambda_{3}, \lambda_{4}, \lambda_{5}\right) \mapsto \\
\left(\zeta \lambda_{1}, \zeta^{4} \lambda_{2}, \zeta^{6} \lambda_{3}, \zeta^{9} \lambda_{4}, \zeta^{14} \lambda_{5}\right) \\
\left(\zeta^{13}=1, \text { primitive }\right)\end{array}$ \\
\hline$W_{24}$ & $\left(t^{4}, t^{9}+\lambda_{1} t^{10}+\lambda_{2} t^{11}+\lambda_{3} t^{15}+\lambda_{4} t^{19}\right)$ & $\begin{array}{c}\mathbb{C}^{4} / G, G=\mathbb{Z} / 13 \mathbb{Z} \\
\left(\lambda_{1}, \lambda_{2}, \lambda_{3}, \lambda_{4}\right) \mapsto \\
\left(\zeta \lambda_{1}, \zeta^{2} \lambda_{2}, \zeta^{6} \lambda_{3}, \zeta^{10} \lambda_{4}\right) \\
\left(\zeta^{13}=1\right)\end{array}$ \\
\hline$W_{30}$ & $\begin{aligned}\left(t^{4}, t^{11}+\lambda_{1} t^{13}+\right. & \lambda_{2} t^{14}+\lambda_{3} t^{17} \\
& \left.+\lambda_{4} t^{21}+\lambda_{5} t^{25}\right)\end{aligned}$ & $\begin{array}{c}\mathbb{C}^{5} / G, G=\mathbb{Z} / 15 \mathbb{Z} \\
\left(\lambda_{1}, \lambda_{2}, \lambda_{3}, \lambda_{4}, \lambda_{5}\right) \mapsto \\
\left(\zeta^{2} \lambda_{1}, \zeta^{5} \lambda_{2}, \zeta^{6} \lambda_{3}, \zeta^{10} \lambda_{4}, \zeta^{14} \lambda_{5}\right) \\
\left(\zeta^{15}=1, \text { primitive }\right)\end{array}$ \\
\hline$W_{2,2 \ell-1}^{\#}$ & $\begin{aligned}\left(t^{4}, t^{10}\right. & +\lambda_{1} t^{2 \ell+9}+\lambda_{2} t^{2 \ell+11}+\lambda_{3} t^{2 \ell+13} \\
& \left.+\lambda_{4} t^{2 \ell+17}+\lambda_{5} t^{2 \ell+21}\right)\left(\lambda_{1} \neq 0\right)\end{aligned}$ & $\begin{array}{c}\left(\mathbb{C}^{*} \times \mathbb{C}^{4}\right) / G, G=\mathbb{Z} / 14 \mathbb{Z} \\
\left(\lambda_{1}, \lambda_{2}, \lambda_{3}, \lambda_{4}, \lambda_{5}\right) \mapsto \\
\left(\zeta^{2 \ell-1} \lambda_{1}, \zeta^{2 \ell+1} \lambda_{2},\right. \\
\left.\zeta^{2 \ell+3} \lambda_{3}, \zeta^{2 \ell+7} \lambda_{4}, \zeta^{2 \ell+11} \lambda_{5}\right) \\
\left(\zeta^{14}=1, \text { primitive }\right)\end{array}$ \\
\hline
\end{tabular}

$$
\begin{gathered}
\left|\begin{array}{cc}
6 \ell_{1} & b \\
6 m_{1} & e
\end{array}\right|+2\left|\begin{array}{cc}
2 h_{1} & h_{2} \\
2 k_{1} & k_{2}
\end{array}\right|+\left|\begin{array}{cc}
a & 2 \ell_{2} \\
c & 2 m_{2}
\end{array}\right|=0, \\
\left|\begin{array}{cc}
2 \ell_{2} & b \\
2 m_{2} & e
\end{array}\right|+\left|\begin{array}{cc}
2 h_{1} & 2 h_{3} \\
2 k_{1} & 2 k_{3}
\end{array}\right|+\left|\begin{array}{cc}
a & 2 \ell_{3} \\
c & 2 m_{3}
\end{array}\right|=0, \\
\left|\begin{array}{cc}
2 \ell_{3} & b \\
2 m_{3} & e
\end{array}\right|+2\left|\begin{array}{cc}
2 h_{2} & h_{3} \\
2 k_{2} & k_{3}
\end{array}\right|+\left|\begin{array}{cc}
a & 6 \ell_{4} \\
c & 6 m_{4}
\end{array}\right|=0, \\
\left|\begin{array}{cc}
24 r_{1} & b \\
24 s_{1} & e
\end{array}\right|+3\left|\begin{array}{cc}
6 \ell_{1} & h_{2} \\
6 m_{1} & k_{2}
\end{array}\right|+3\left|\begin{array}{cc}
2 h_{1} & 2 \ell_{2} \\
2 k_{1} & 2 m_{2}
\end{array}\right|+\left|\begin{array}{cc}
a & 6 r_{2} \\
c & 6 s_{2}
\end{array}\right|=0 .
\end{gathered}
$$

Next, we have the symplectic rigidity: 
Proposition 4.3. Let $f_{\lambda}$ and $f_{\lambda^{\prime}}$ be germs belonging to one of the symplectic normal forms of simple or uni-modal parametric plane curve singularities. If $f_{\lambda}$ and $f_{\lambda^{\prime}}$ are symplectomorphic, then they are linearly symplectomorphic: If there exists a symplectomorphic equivalence $(\sigma, \tau)$ satisfying $\tau \circ f_{\lambda^{\prime}}=f_{\lambda} \circ \sigma$, then there exists a symplectomorphic equivalence $(\Sigma, T)$ such that $T \circ f_{\lambda^{\prime}}=f_{\lambda} \circ \Sigma, \Sigma:(\mathbb{C}, 0) \rightarrow(\mathbb{C}, 0)$ is a complex linear transformation, and $T:\left(\mathbb{C}^{2}, 0\right) \rightarrow\left(\mathbb{C}^{2}, 0\right)$ is a complex linear symplectic transformation.

Proof. We give the calculation in the case of $W_{30}$. Other cases can be treated similarly.

Set $f_{\lambda}=\left(t^{4}, t^{11}+\lambda_{1} t^{13}+\lambda_{2} t^{14}+\lambda_{3} t^{17}+\lambda_{4} t^{21}+\lambda_{5} t^{25}\right)$, and suppose $f_{\lambda}$ and $f_{\lambda^{\prime}}$ are symplectomorphic for $\lambda=\left(\lambda_{1}, \ldots, \lambda_{5}\right)$ and $\lambda^{\prime}=\left(\lambda_{1}^{\prime}, \ldots, \lambda_{5}^{\prime}\right)$.

Set $\sigma(t)=a_{1} t+a_{2} t^{2}+\cdots$ and, as components of $\tau(x, y)$,

$$
\begin{aligned}
x \circ \tau(x, y)= & a x+b y+h_{1} x^{2}+h_{2} x y+h_{3} y^{2}+\ell_{1} x^{3}+\ell_{2} x^{2} y+\ell_{3} x y^{2} \\
& +\ell_{4} y^{3}+\cdots, \\
y \circ \tau(x, y)= & c x+e y+k_{1} x^{2}+k_{2} x y+k_{3} y^{2}+m_{1} x^{3}+m_{2} x^{2} y+m_{3} x y^{2} \\
& +m_{4} y^{3}+\cdots .
\end{aligned}
$$

Consider the equation $f_{\lambda}(\sigma(t))=\tau\left(f_{\lambda^{\prime}}(t)\right)$ :

$$
\begin{aligned}
& \sigma(t)^{4}=x \circ \tau\left(t^{4}, t^{11}+\lambda_{1}^{\prime} t^{13}+\lambda_{2}^{\prime} t^{14}+\lambda_{3}^{\prime} t^{17}+\lambda_{4}^{\prime} t^{21}+\lambda_{5}^{\prime} t^{25}\right), \\
& \sigma(t)^{11}+\lambda_{1} \sigma(t)^{13}+\lambda_{2} \sigma(t)^{14}+\lambda_{3} \sigma(t)^{17}+\lambda_{4} \sigma(t)^{21}+\lambda_{5} \sigma(t)^{25} \\
& =y \circ \tau\left(t^{4}, t^{11}+\lambda_{1}^{\prime} t^{13}+\lambda_{2}^{\prime} t^{14}+\lambda_{3}^{\prime} t^{17}+\lambda_{4}^{\prime} t^{21}+\lambda_{5}^{\prime} t^{25}\right) .
\end{aligned}
$$

Now we are going to determine the coefficients of $\sigma$ and $\tau$ of lower degree terms, using the equations $(*)$ and $(* *)$ in a zigzag manner. We denote the terms of degree $i$ of both sides in $(*)$ (resp. $(* *))$ by $(* i)$ (resp. $(* * i)$ ). First by $\left({ }^{*} 4\right)$, we have $a_{1}^{4}=a$. By $\left({ }^{*} 5\right),\left({ }^{*} 6\right),\left({ }^{*} 7\right), a_{2}=0, a_{3}=0, a_{4}=0$. By $\left(*^{*} 4\right)$, $c=0$. By $(* * 8), k_{1}=0$. By (**11), $a_{1}^{11}=e$. Since $\tau$ is a symplectomorphism, we see that $a e=1$, by Lemma 4.2 , so $a_{1}^{15}=1$. By $\left({ }^{* *} 12\right), m_{1}=0$. By $\left({ }^{* *} 13\right)$, $\lambda_{1} a^{13}=e \lambda_{1}^{\prime}$ and therefore $\lambda_{1} a^{2}=\lambda_{1}^{\prime}$. By (**14), $\lambda_{2} a^{14}=e \lambda_{2}^{\prime}$ and therefore $\lambda_{1} a^{3}=\lambda_{1}^{\prime}$. By $(* * 15), 11 a_{1}^{10} a_{5}=k_{2}$. From $\left({ }^{*} 8\right), 4 a_{3} a_{5}=h_{1}$. Since $\tau$ is a symplectomorphism, we have $2 h_{1} e+a k_{2}=0$, by Lemma 4.2. Thus we see that $a_{5}=0$. Then $k_{2}=0, h_{1}=0$. By $(* 9), 4 a_{3} a_{6}=0$ so $a_{6}=0$. By $\left({ }^{*} 10\right)$, $a_{7}=0$. By $\left({ }^{*} 11\right), 4 a_{1}^{3} a_{8}=b$. Then by (**17), $\lambda_{3} a^{17}=e \lambda_{3}^{\prime}$, thus $\lambda_{3} a^{6}=\lambda_{3}^{\prime}$. By $\left({ }^{* * 18}\right), a_{8}=0$. Therefore $b=0$. By $\left({ }^{*} 12\right), 4 a_{1}^{3} a_{8}=\ell_{1}$. By $\left({ }^{* * 19}\right)$, $11 a_{1}^{10} a_{9}=m_{2}$. Since $\tau$ is a symplectomorphism, we have $6 \ell_{1} e+2 a m_{2}=0$ by Lemma 4.2. Thus $a_{9}=0$. Then $\ell_{1}=0, m_{2}=0$. By $\left({ }^{*} 13\right)-\left({ }^{*} 14\right), a_{10}=0$, $a_{11}=0$. By $\left({ }^{* *} 21\right), \lambda_{4} a^{21}=e \lambda_{4}^{\prime}$ so $\lambda_{4} a^{10}=\lambda_{4}^{\prime}$. By $\left({ }^{* *} 22\right), 11 a_{1}^{10} a_{12}=k_{3}$. By (*15), $4 a_{1}^{3} a_{12}=h_{2}$. Since $\tau$ is a symplectomorphism, we have $h_{2} e+2 a k_{3}=0$ by Lemma 4.2. Therefore $a_{12}=0$, and $k_{3}=0, h_{2}=0$. Then, by $(* * 23)$, $a_{13}=0$, and by $\left(*^{*} 17\right)-\left({ }^{*} 18\right), a_{14}=0, a_{15}=0$. Finally, by $\left({ }^{* *} 25\right), \lambda_{5} a^{25}=$ 
$e \lambda_{5}^{\prime}$, and $\lambda_{5} a^{13}=\lambda_{5}^{\prime}$. Therefore, defining $T$ and $\Sigma$ to be the linear parts of $\tau$ and $\sigma$ respectively, we have $T \circ f_{\lambda^{\prime}}=f_{\lambda} \circ \Sigma$.

Proof of Theorem 4.1. Once the rigidity as in Proposition 4.3 is established, it suffices just to observe that $T \circ f_{\lambda^{\prime}}=f_{\lambda} \circ \Sigma$ for a linear transformation $\Sigma(t)=a_{1} t$ and a symplectic linear transformation $T(x, y)=$ $(a x+b y, c x+e y)$. Then we easily see that $a_{1}^{m}=a, c=0, a_{1}^{n}=e$. Since $T \in \operatorname{SL}(2, \mathbb{C})$ we see $a_{1}^{m+n}=1$. Then, setting $\zeta=a_{1}$, we obtain Theorem 4.1 .

REMARK 4.4. If two curve-germs $f, g:(\mathbb{C}, 0) \rightarrow\left(\mathbb{C}^{2}, 0\right)$ are symplectomorphic, then they are symplectically isotopic, that is, there exist $C^{\infty}$ families of bi-holomorphic diffeomorphisms $\sigma_{s}$ and bi-holomorphic symplectomorphisms $\tau_{s}(s \in[0,1])$ on $(\mathbb{C}, 0)$ and $\left(\mathbb{C}^{2}, 0\right)$ respectively such that $\sigma_{0}(t)=t$, $\tau_{0}(x, y)=(x, y)$ and $\tau_{1}(g(t))=f\left(\sigma_{1}(t)\right)$. This is a feature of the complex case and it is proved by using the fact that $\mathrm{SL}(2, \mathbb{C})$ is arc-wise connected and the group of symplectomorphisms with identity linear part is arc-wise connected (cf. [14]). Thus our symplectic moduli spaces in Tables 1 and 2 are also moduli spaces for symplectic isotopy equivalence (cf. [30]).

5. Differential normal forms. The classification of simple singularities by Bruce-Gaffney [3] is extended to the following:

THEOREM 5.1. Under diffeomorphic equivalence the simple and uni-modal singularities of parametric plane curves $f:(\mathbb{C}, 0) \rightarrow\left(\mathbb{C}^{2}, 0\right)$ are completely classified into the following list:

$$
\begin{array}{ll}
A_{2 \ell}: & \left(t^{2}, t^{2 \ell+1}\right)(\ell=1,2,3, \ldots), \\
E_{6 \ell}: \quad & \left(t^{3}, t^{3 \ell+1}+t^{3(\ell+p)+2}\right)(0 \leq p \leq \ell-2),\left(t^{3}, t^{3 \ell+1}\right), \\
E_{6 \ell+2}: & \left(t^{3}, t^{3 \ell+2}+t^{3(\ell+p)+4}\right)(0 \leq p \leq \ell-2),\left(t^{3}, t^{3 \ell+2}\right), \\
W_{12}: \quad & \left(t^{4}, t^{5}+t^{7}\right),\left(t^{4}, t^{5}\right), \\
W_{18}: \quad & \left(t^{4}, t^{7}+t^{9}\right),\left(t^{4}, t^{7}+t^{13}\right),\left(t^{4}, t^{7}\right), \\
W_{1,2 \ell-1}^{\#}: & \left(t^{4}, t^{6}+t^{2 \ell+5}\right), \\
N_{20}: \quad & \left(t^{5}, t^{6}+t^{8}+\lambda t^{9}\right)(-\lambda \sim \lambda),\left(t^{5}, t^{6}+t^{9}\right),\left(t^{5}, t^{6}+t^{14}\right),\left(t^{5}, t^{6}\right), \\
N_{24}: \quad & \left(t^{5}, t^{7}+t^{8}+\lambda t^{11}\right),\left(t^{5}, t^{7}+t^{11}+\lambda t^{13}\right)(-\lambda \sim \lambda), \\
& \left(t^{5}, t^{7}+t^{13}\right),\left(t^{5}, t^{7}+t^{18}\right),\left(t^{5}, t^{7}\right), \\
N_{28}: \quad & \left(t^{5}, t^{8}+t^{9}+\lambda t^{12}\right),\left(t^{5}, t^{8}+t^{12}+\lambda t^{14}\right)(-\lambda \sim \lambda), \\
& \left(t^{5}, t^{8}+t^{14}+\lambda t^{17}\right)(-\lambda \sim \lambda),\left(t^{5}, t^{8}+t^{17}\right),\left(t^{5}, t^{8}+t^{22}\right), \quad\left(t^{5}, t^{8}\right),
\end{array}
$$




$$
\begin{array}{ll}
W_{24}: \quad & \left(t^{4}, t^{9}+t^{10}+\lambda t^{11}\right)\left(\lambda \neq \frac{19}{18}\right),\left(t^{4}, t^{9}+t^{10}+\frac{19}{18} t^{11}+\lambda t^{15}\right), \\
& \left(t^{4}, t^{9}+t^{11}\right),\left(t^{4}, t^{9}+t^{15}\right),\left(t^{4}, t^{9}+t^{19}\right),\left(t^{4}, t^{9}\right), \\
W_{30}: \quad & \left(t^{4}, t^{11}+t^{13}+\lambda t^{14}\right)(-\lambda \sim \lambda),\left(t^{4}, t^{11}+t^{14}+\lambda t^{17}\right)\left(\lambda \neq \frac{25}{22}\right), \\
& \left(t^{4}, t^{11}+t^{14}+\frac{25}{22} t^{17}+\lambda t^{21}\right)\left(\omega \lambda \sim \lambda, \omega^{3}=1\right), \\
& \left(t^{4}, t^{11}+t^{17}\right),\left(t^{4}, t^{11}+t^{21}\right),\left(t^{4}, t^{11}+t^{25}\right),\left(t^{4}, t^{11}\right), \\
W_{2,2 \ell-1}^{\#}: & \left(t^{4}, t^{10}+t^{2 \ell+9}+\lambda t^{2 \ell+11}\right)\left(\omega \lambda \sim \lambda, \omega^{2 \ell-1}=1\right)(\ell=1,2,3, \ldots) .
\end{array}
$$

In the list, for instance $-\lambda \sim \lambda$ means that $\left(t^{5}, t^{6}+t^{8}+\lambda^{\prime} t^{9}\right)$ is diffeomorphic to $\left(t^{5}, t^{6}+t^{8}+\lambda t^{9}\right)$ if and only if $\lambda^{\prime}= \pm \lambda$.

REMARK 5.2. Ebey [7] gave diffeomorphic classifications of the cases $(2,2 \ell+1),(3,3 \ell+1),(3,3 \ell+2),(4,5),(4,6,2 \ell+5),(4,7)$ and $(5,9)$. Also he classified the cases $(4,9),(4,10,2 \ell+9)$ and $(4,11)$, but his classification has several omissions and errors, which are corrected in our classification. Note moreover that several cases with modality $\geq 2$ are classified under diffeomorphisms by several authors: $(5,9)([7]) ;(6,7)([34]) ;(5,11)([21])$; $(2 p, 2 q, 2 q+\ell)([23]) ;(6,9,10)([11])$.

Proof of Theorem 5.1: Theorem 5.1 can be established by a similar method to the one in [3]. However, we note that the symplectic normal forms (Theorem 3.10 can play the role of an intermediate classification, which also makes the diffeomorphic classification easier and clearer.

Theorem 5.1 will be proved if we classify all remaining cases of Lemma 2.2

Here we will treat only the classes $N_{20}$ and $W_{30}$ with Puiseux characteristic $(5,6)$ and $(4,11)$ respectively. The remaining cases can be treated by the same method.

We start with the symplectic normal form

$$
f_{\lambda}(t)=\left(t^{5}, t^{6}+\lambda_{1} t^{8}+\lambda_{2} t^{9}+\lambda_{3} t^{14}\right),
$$

of class $N_{20}$. Consider, for a given vector field $v(t)=(0, \rho(t))$, Mather's equation

$$
\left(\begin{array}{c}
0 \\
\rho(t)
\end{array}\right)=\xi\left(\begin{array}{c}
5 t^{4} \\
6 t^{5}+8 \lambda_{1} t^{7}+\cdots
\end{array}\right)+\left(\begin{array}{c}
\eta_{1}\left(f_{\lambda}(t)\right) \\
\eta_{2}\left(f_{\lambda}(t)\right)
\end{array}\right),
$$

and try to find $\xi(t), \eta_{1}(x, y), \eta_{2}(x, y)$ with $\xi(0)=0, \eta_{1}(0,0)=0, \eta_{2}(0,0)=0$. We can take $N^{\prime}=20$ in the notation of Lemma 3.7(1), so, by Lemma 3.7 (2), $v=(0, \rho(t))$ is symplectically solvable if $\operatorname{ord}(\rho) \geq 15$.

If $\lambda_{1} \neq 0$, then $v=\left(0, t^{k}\right)$ is solvable up to higher order terms for $k=8$, $10,11,12,13,14$. Thus we reduce our family to $f_{\lambda}(t)=\left(t^{5}, t^{6}+t^{8}+\lambda t^{9}\right)$, $\lambda \in \mathbb{C}$, by diffeomorphisms. By further calculations on formal power series, we see that $f_{\lambda}$ and $f_{\lambda^{\prime}}$ are diffeomorphic if and only if $\lambda^{\prime}= \pm \lambda$. 
If $\lambda_{1}=0, \lambda_{2} \neq 0$, then $v=\left(0, t^{k}\right)$ is solvable up to higher order terms for $k=9,10,11,12,13,14$, and we have the differential normal form $\left(t^{5}, t^{6}+t^{9}\right)$.

If $\lambda_{1}=0, \lambda_{2}=0, \lambda_{3} \neq 0$, then $v=\left(0, t^{14}\right)$ is solvable, and we get the differential normal form $\left(t^{5}, t^{6}+t^{14}\right)$.

Lastly if $\lambda_{1}=0, \lambda_{2}=0, \lambda_{3}=0$, then $\left(t^{5}, t^{6}\right)$ is regarded as the differential normal form.

For the case $W_{30}$, consider the symplectic normal form

$$
f_{\lambda}(t)=\left(t^{4}, t^{11}+\lambda_{1} t^{13}+\lambda_{2} t^{14}+\lambda_{3} t^{17}+\lambda_{4} t^{21}+\lambda_{5} t^{25}\right) .
$$

Consider Mather's equation

$$
\left(\begin{array}{c}
0 \\
\rho(t)
\end{array}\right)=\xi\left(\begin{array}{c}
4 t^{3} \\
11 t^{10}+13 \lambda_{1} t^{12}+\cdots
\end{array}\right)+\left(\begin{array}{l}
\eta_{1}\left(f_{\lambda}(t)\right) \\
\eta_{2}\left(f_{\lambda}(t)\right)
\end{array}\right) .
$$

We can take $N^{\prime}=30$ in this case so $v(t)=(0, \rho(t))$ is symplectically solvable if $\operatorname{ord}(\rho) \geq 26$. Suppose $\lambda_{1} \neq 0$. Then we see $v(t)=\left(0, t^{k}\right)$ is solvable for $k=13$, up to higher order terms, and solvable for any $k \geq 15$. Then, by the homotopy method, we see that, if $\lambda_{1} \neq 0$, then $f$ is diffeomorphic to $\left(t^{4}, t^{11}+t^{13}+\lambda t^{14}\right)$ for some $\lambda \in \mathbb{C}$. If $\lambda_{1}=0, \lambda_{2} \neq 0$, then $f$ is diffeomorphic to $\left(t^{4}, t^{11}+t^{14}+\lambda t^{17}\right)$ for some $\lambda \in \mathbb{C}, \lambda \neq \frac{25}{22}$, or to $\left(t^{4}, t^{11}+t^{14}+\frac{25}{22} t^{17}+\lambda t^{21}\right)$ for some $\lambda \in \mathbb{C}$. If $\lambda_{1}=0, \lambda_{2}=0, \lambda_{3} \neq 0$ (resp. $\lambda_{1}=0, \lambda_{2}=0, \lambda_{3}=0$, $\left.\lambda_{4} \neq 0 ; \lambda_{1}=0, \lambda_{2}=0, \lambda_{3}=0, \lambda_{4}=0, \lambda_{5} \neq 0\right)$, then $f$ is diffeomorphic to $\left(t^{4}, t^{11}+t^{17}\right)$ (resp. $\left.\left(t^{4}, t^{11}+t^{21}\right) ;\left(t^{4}, t^{11}+t^{25}\right)\right)$. The exact determination of the differential moduli space is completed just by straightforward formal calculations.

REMARK 5.3. In general, for each equi-singularity class, the symplectic moduli space is mapped canonically onto the differential moduli space, i.e. the ordinary moduli space. The dimension of the fiber over a diffeomorphism class $[f]$ is called the symplectic defect and denoted by $\operatorname{sd}(f)$ in [15]. It is known that $\operatorname{sd}(f)=\mu(f)-\tau(f)$, where $\mu(f)=2 \delta(f)$ is the Milnor number of $f$ and $\tau(f)$ is the Tyurina number of $f([30$, [20], [5]). Let $s(f)$ (resp. $c(f))$ be the symplectic modality, that is, the number of parameters in the symplectic normal form of $f$ (resp. the codimension of the locus in the parameter space corresponding to germs diffeomorphic to $f)$. Then $s(f)-c(f)=\operatorname{sd}(f)$. Thus we have the following formula for the Tyurina number (by means of Varchenko-Lando's formula):

$$
\tau(f)=2 \delta(f)+c(f)-s(f) .
$$

For example, for $f=\left(t^{4}, t^{11}+t^{21}\right)$ in the case of $W_{30}$, we have $\delta(f)=$ $15, c(f)=3, s(f)=5$ and in fact $\tau(f)=28$.

Note that the differential moduli space is not a Hausdorff space, while the symplectic moduli space is, at least for 0 -modal and 1-modal cases, as 
we clearly observe in Theorems 5.1 and 4.1 . Therefore the symplectic moduli space can be called a Hausdorffication of the differential moduli space.

REMARK 5.4. The adjacency of simple and uni-modal singularities of parametric plane curves is generated (as an ordering) by $A_{2 \ell} \leftarrow A_{2 \ell+2}, E_{6 \ell} \leftarrow$ $E_{6 \ell+2} \leftarrow E_{6 \ell+6}(\ell=1,2, \ldots), A_{6 s-2} \leftarrow E_{12 s-6}, A_{6 s} \leftarrow E_{12 s}, A_{6 s-2} \leftarrow$ $E_{12 s-4}, A_{6 s+2} \leftarrow E_{12 s+2}(s=1,2, \ldots), E_{8} \leftarrow W_{12} \leftarrow W_{18}, W_{12} \leftarrow W_{1,1}^{\#}$, $E_{12} \leftarrow W_{1,1}^{\#} \leftarrow W_{18}, W_{1,2 \ell-1}^{\#} \leftarrow W_{1,2 \ell+1}^{\#}(\ell=1,2, \ldots), W_{1,1}^{\#} \leftarrow N_{20} \leftarrow$ $N_{24} \leftarrow N_{28}, W_{18} \leftarrow N_{24}, W_{24} \leftarrow N_{28}, W_{18} \leftarrow W_{24} \leftarrow W_{30}, E_{18} \leftarrow W_{24} \leftarrow$ $W_{2,1}^{\#}, E_{20} \leftarrow W_{30}, W_{2,2 \ell-1}^{\#} \leftarrow W_{2,2 \ell+1}^{\#}(\ell=1,2, \ldots)$.

REMARK 5.5 (Classification of curves with characteristic $(6,7)$ ). Let $f$ : $(\mathbb{C}, 0) \rightarrow\left(\mathbb{C}^{2}, 0\right)$ be a plane branch of characteristic $(6,7), m=6, \beta_{1}=7$. The quotient $\mathcal{O}_{1} / f^{*} \mathcal{O}_{2}$ has the monomial basis

$$
t, t^{2}, t^{3}, t^{4}, t^{5}, t^{8}, t^{9}, t^{10}, t^{11}, t^{15}, t^{16}, t^{17}, t^{22}, t^{23}, t^{29} .
$$

The symplectic normal form is given by

$$
f_{\lambda}(t)=\left(t^{6}, t^{7}+\lambda_{1} t^{9}+\lambda_{2} t^{10}+\lambda_{3} t^{11}+\lambda_{4} t^{16}+\lambda_{5} t^{17}+\lambda_{6} t^{23}\right),
$$

$\left(\lambda_{1}, \lambda_{2}, \lambda_{3}, \lambda_{4}, \lambda_{5}, \lambda_{6}\right) \in \mathbb{C}^{6}$. Moreover we can check that $f_{\lambda}$ and $f_{\lambda^{\prime}}$ are symplectomorphic if and only if there exists a $\zeta \in \mathbb{C}$ with $\zeta^{13}=1$ satisfying $\lambda_{1}^{\prime}=\zeta^{2} \lambda_{1}, \quad \lambda_{2}^{\prime}=\zeta^{3} \lambda_{2}, \quad \lambda_{3}^{\prime}=\zeta^{4} \lambda_{3}, \quad \lambda_{4}^{\prime}=\zeta^{9} \lambda_{4}, \quad \lambda_{5}^{\prime}=\zeta^{10} \lambda_{5}, \quad \lambda_{6}^{\prime}=\zeta^{16} \lambda_{6}$.

Thus the symplectic moduli space $\mathcal{M}_{\text {symp }}(6,7)$ is homeomorphic to $\mathbb{C}^{6} / G$ for $G=\mathbb{Z} / 13 \mathbb{Z}$ with the representation $G \rightarrow \mathrm{GL}(6, \mathbb{C})$ given by

$$
\zeta \mapsto\left(\left(\lambda_{1}, \lambda_{2}, \lambda_{3}, \lambda_{4}, \lambda_{5}, \lambda_{6}\right) \mapsto\left(\zeta^{2} \lambda_{1}, \zeta^{3} \lambda_{2}, \zeta^{4} \lambda_{3}, \zeta^{9} \lambda_{4}, \zeta^{10} \lambda_{5}, \zeta^{16} \lambda_{6}\right)\right) .
$$

As a by-product we get an exact diffeomorphic classification of plane curves of characteristic $(6,7)$ due to Zariski using our symplectic method:

Theorem 5.6 (Zariski [34]). Any plane curve-germ $f:(\mathbb{C}, 0) \rightarrow\left(\mathbb{C}^{2}, 0\right)$ of Puiseux characteristic $(6,7)$ is diffeomorphic to one of the following normal forms:

$$
\begin{array}{ll}
Z_{\lambda, \mu}^{0}: & \left(t^{6}, t^{7}+t^{9}+\lambda t^{10}+\mu t^{11}\right), \mu \neq \frac{9}{8} \lambda^{2}+\frac{23}{14}, \quad( \pm \lambda, \mu) \sim(\lambda, \mu), \\
Z_{\lambda, \nu}^{1}: & \left(t^{6}, t^{7}+t^{9}+\lambda t^{10}+\left(\frac{9}{8} \lambda^{2}+\frac{23}{14}\right) t^{11}+\nu t^{17}\right), \quad( \pm \lambda, \nu) \sim(\lambda, \nu), \\
Z_{\lambda}^{\prime 1}: & \left(t^{6}, t^{7}+t^{10}+\lambda t^{11}\right), \omega \lambda \sim \lambda, \omega^{3}=1, \\
Z_{\lambda}^{2}: & \left(t^{6}, t^{7}+t^{11}+\lambda t^{16}\right), \omega \lambda \sim \lambda, \omega^{4}=1, \\
Z_{\lambda}^{3}: & \left(t^{6}, t^{7}+t^{16}+\lambda t^{17}\right), \omega \lambda \sim \lambda, \omega^{9}=1, \\
Z^{4}: & \left(t^{6}, t^{7}+t^{17}\right), \\
Z^{5}: & \left(t^{6}, t^{7}+t^{23}\right), \\
Z^{6}: & \left(t^{6}, t^{7}\right) .
\end{array}
$$


Acknowledgements. This paper is an extended version of the preprint [17. The authors thank T. Krasiński for valuable comments, in particular, for the information on the reference [34]. They also thank the referees for their helpful comments. Research of the first author was supported by Grants-inAid for Scientific Research, No. 14340020, and research of the second author was partly supported by Grant MNiSzW No. 201397237.

\section{References}

[1] V. I. Arnold, Local normal forms of functions, Invent. Math. 35 (1976), 87-109.

[2] - First steps of local symplectic algebra, in: Differential Topology, Infinite-Dimensional Lie Algebras, and Applications, Amer. Math. Soc. Transl. (2) 194, Amer. Math. Soc., Providence, RI, 1999, 1-8.

[3] J. W. Bruce and T. Gaffney, Simple singularities of mappings $\mathbb{C}, 0 \rightarrow \mathbb{C}^{2}, 0$, J. London Math. Soc. 26 (1982), 465-474.

[4] J. Chądzyński and A. Płoski, An inequality for intersection multiplicity of analytic curves, Bull. Polish Acad. Sci. Math. 36 (1988), 113-117.

[5] W. Domitrz, S. Janeczko and M. Zhitomirskii, Relative Poincaré lemma, contractibility, quasi-homogeneity and vector fields tangent to a singular variety, Illinois J. Math. 48 (2004), 803-835.

[6] W. Domitrz and J. H. Rieger, Volume-preserving diffeomorphisms on varieties and $\mathcal{A}_{\Omega}$-equivalence of maps, preprint, 2006.

[7] S. Ebey, The classification of singular points of algebraic curves, Trans. Amer. Math. Soc. 118 (1965), 454-471.

[8] M. D. Garay, An isochore versal deformation theorem, Topology 43 (2004), 10811088.

[9] E. Garcia Barroso, T. Krasiński and A. Płoski, The Eojasiewicz numbers and plane curve singularities, Ann. Polon. Math. 87 (2005), 127-150.

[10] A. B. Givental', Singular Lagrangian manifolds and their Lagrangian mappings, Itogi Nauki Tekh. Ser. Sovrem. Prob. Mat. 33, VINITI, 1988, 55-112; English transl.: J. Soviet Math. 52 (1990), 3246-3278.

[11] A. Hefez and M. E. Hernandes, Classification of algebroid plane curves with semigroup $\langle 6,9,19\rangle$, Comm. Algebra 31 (2003), 3847-3861.

[12] -, 一, The analytic classification of plane branches, arXiv:0707.4502.

[13] -, -, Analytic classification of plane branches up to multiplicity 4, J. Symbolic Comput. 44 (2009), 626-634.

[14] G. Ishikawa, Determinacy, transversality and Lagrange stability, in: Geometry and Topology of Caustics - CAUSTICS '98, Banach Center Publ. 50, Inst. Math., Polish Acad. Sci., 1999, 123-135.

[15] G. Ishikawa and S. Janeczko, Symplectic bifurcations of plane curves and isotropic liftings, Quart. J. Math. 54 (2003), 73-102.

[16] - - - Symplectic singularities of isotropic mappings, in: Geometric Singularity Theory, Banach Center Publ. 65, Inst. Math., Polish Acad. Sci., 2004, 85-106.

[17] - - - The complex symplectic moduli spaces of uni-modal parametric plane curve singularities, preprint 664, Inst. Math., Polish Acad. Sci., 2006.

[18] - - - Symplectic invariants of parametric singularities, preprint 704, Inst. Math., Polish Acad. Sci., 2009. 
[19] P. A. Kolgushkin, Classification of simple multigerms of curves in a space endowed with a symplectic structure, St. Petersburg Math. J. 15 (2004), 103-126.

[20] S. K. Lando, Normal forms of the degrees of a volume form, Funct. Anal. Appl. 19 (1984), 146-148.

[21] O. A. Laudal and G. Pfister, Local Moduli and Singularities, Lecture Notes in Math. 1310, Springer, Berlin, 1988.

[22] Lê Dũng Tráng, Sur un critère d'équisingularité, C. R. Acad. Sci. Paris Sér. A 272 (1971), 138-140.

[23] I. Luengo and G. Pfister, Normal forms and moduli spaces of curve singularities with semigroup $\langle 2 p, 2 q, 2 p q+d\rangle$, Compos. Math. 76 (1990), 247-264.

[24] J. N. Mather, Stability of $C^{\infty}$ mappings III: Finitely determined map-germs, Publ. Math. I.H.E.S. 35 (1968), 127-156.

[25] J. Milnor, Singular Points of Complex Hypersurfaces, Ann. of Math. Stud. 61, Princeton Univ. Press, 1986.

[26] A. Płoski, On special values for pencils of plane curve singularities, Univ. Iagel. Acta Math. 42 (2004), 7-13.

[27] B. Teissier, Déformation à type topologique constant, Astérisque 16 (1973).

[28] —, Cycles évanescents, sections planes, et conditions de Whitney, Astérisque 7-8 (1973).

[29] -, Appendix in 34].

[30] A. N. Varchenko, Local classification of volume forms in the presence of a hypersurface, Funct. Anal. Appl. 19 (1984), 269-276.

[31] J. M. Wahl, Equisingular deformations of plane algebroid curves, Trans. Amer. Math. Soc. 193 (1974), 143-170.

[32] C. T. C. Wall, Finite determinacy of smooth map-germs, Bull. London Math. Soc. 13 (1981), 481-539.

[33] - Singular Points of Plane Curves, Cambridge Univ. Press, 2004.

[34] O. Zariski, Le problème des modules pour les branches planes, École Polytechnique, Paris, 1973, and Hermann, Paris, 1986; English transl.: The Moduli Problem for Plane Branches, Univ. Lecture Ser. 39, Amer. Math. Soc., 2007.

Goo Ishikawa

Department of Mathematics

Hokkaido University

Sapporo 060-0810, Japan

E-mail: ishikawa@math.sci.hokudai.ac.jp
Stanisław Janeczko Institute of Mathematics Polish Academy of Sciences Sniadeckich 8

00-956 Warszawa, Poland E-mail: janeczko@impan.pl and

Faculty of Mathematics and Information Science Warsaw University of Technology Pl. Politechniki 1 00-661 Warszawa, Poland

Received 12.11.2009

and in final form 10.3.2010 